\title{
Nonlinear Sum Operator Equations with a Parameter and Application to Second-Order Three-Point BVPs
}

\author{
Wen-Xia Wang, ${ }^{1}$ Xi-Lan Liu, ${ }^{2}$ and Piao-Piao Shi ${ }^{3}$ \\ ${ }^{1}$ Department of Mathematics, Taiyuan Normal University, Taiyuan 030012, China \\ ${ }^{2}$ Department of Mathematics and Statistics, Qinghai Nationalities University, Xining 810007, China \\ ${ }^{3}$ School of Mathematics, Jinzhong University, Jinzhong 030600, China
}

Correspondence should be addressed to Wen-Xia Wang; wwxgg@126.com

Received 29 November 2013; Revised 25 January 2014; Accepted 27 January 2014; Published 16 March 2014

Academic Editor: Erdal Karapınar

Copyright (C) 2014 Wen-Xia Wang et al. This is an open access article distributed under the Creative Commons Attribution License, which permits unrestricted use, distribution, and reproduction in any medium, provided the original work is properly cited.

\begin{abstract}
A class of nonlinear sum operator equations with a parameter on order Banach spaces were considered. The existence and uniqueness of positive solutions for this kind of operator equations and the dependence of solutions on the parameter have been obtained by using the properties of cone and nonlinear analysis methods. The critical value of the parameter was estimated. Further, the application to some nonlinear three-point boundary value problems was given to show the significance of the discussion.
\end{abstract}

\section{Introduction and Preliminaries}

The aim of this paper is to investigate the existence and uniqueness of positive solution for the following operator equations:

$$
L(\lambda, x)=x,
$$

where $L(\lambda, x)=A x+\lambda B x, A$ is an operator with concavity, $B$ is a pseudo subhomogeneous operator, and $\lambda$ is a parameter. In addition, by applying our results to the second-order threepoint boundary value problem (BVP),

$$
\begin{gathered}
-x^{\prime \prime}(t)=\lambda f(t, x(t))+g(t), \quad t \in(0,1), \\
x(0)=\tau x^{\prime}(0), \quad x(1)-\sigma x(\xi)=\int_{0}^{1} h(x(s)) d s,
\end{gathered}
$$

where $f \in C([0,1] \times R), g \in C[0,1], h \in C(R), \tau \geq$ $0,0<\xi<1$, and $0<\sigma \leq 1$; we obtain that there exists a $\lambda^{*}>0$, such that $\operatorname{BVP}(2)$ has a unique positive solution and no positive solution for $0<\lambda<\lambda^{*}$ and $\lambda \geq \lambda^{*}$, respectively. In particular, such a positive solution $x_{\lambda}(t)$ of $\mathrm{BVP}(2)$ is increasing and continuous in $\lambda$ for $\lambda \in\left[0, \lambda^{*}\right)$ and $\lim _{\lambda \rightarrow \lambda^{*}-0}\left\|x_{\lambda}\right\|_{c}=+\infty$. Further, we estimate the critical value $\lambda^{*}$.
In recent years, many authors focus on multipoint boundary value problems for differential equations, since these problems arise in a variety of different areas of applied mathematics and physics (see [1]). For example, by using degreetheoretic arguments, Gupta [2] obtained the existence and uniqueness theorems for the following three-point boundary value problem:

$$
\begin{gathered}
x^{\prime \prime}(t)=f\left(t, x(t), x^{\prime}(t)\right)-e(t), \quad t \in(0,1), \\
x(0)=0, \quad x(\xi)=x(1) .
\end{gathered}
$$

Since then, the existence of solutions for nonlinear multipoint boundary value problems has been studied by many authors (see [3-8] and their references). The cases with special boundary value conditions in $\mathrm{BVP}(2)$ were discussed by [27], where $\tau=0$ and $h(x)=0$ or $\tau=0, \sigma=1$ and $h(x)=$ 0 . However, to the best of our knowledge, little has been done for the multipoint boundary value problem (2) with parameter, perturbed loading force, and nonlinear boundary conditions, especially on the existence and uniqueness of positive solution and the dependence of solutions on the parameter $\lambda$.

It is well known that fixed point theory is an effective tool in the treatment of existence results of boundary value problems for nonlinear differential equations. Many researchers 
were concerned with the existence and uniqueness of fixed point for concave operators and sum operators. For example, [9-15] investigated eigenvalue problems of concave operators, the existence and uniqueness of positive fixed point for concave operators, and the existence and uniqueness of positive fixed point for sum operator, respectively. However, to our knowledge, few of the results in literature can be applied to $\operatorname{BVP}(2)$ successfully. The above reasons stimulate us to do this work.

First, we consider the existence and property of positive solutions for nonlinear operator equations (1) on order Banach space $E$.

To be clear, some definitions, notations, and lemmas are presented as follows.

Let $E$ be a real Banach space which is partially ordered by a cone $P$ of $E$, that is, $x \leq y$ if and only if $y-x \in P$, and let $\theta$ be the zero element of $E$. If $x \leq y$ and $x \neq y$, then we denote $x<y$ or $y>x$. A nonempty closed convex set $P \subset E$ is a cone if it satisfies (i) $x \in P, r \geq 0 \Rightarrow r x \in P$; (ii) $x \in P,-x \in P \Rightarrow x=\theta$. A cone $P$ is said to be normal if there exists a positive number $N$, called the normal constant of $P$, such that, for all $x, y \in E, \theta \leq x \leq y$ implies $\|x\| \leq N\|y\|$.

Let $D \subseteq E$. An operator $T: D \rightarrow E$ is said to be increasing if, for $x_{1}, x_{2} \in D, x_{1} \leq x_{2} \Rightarrow T x_{1} \leq T x_{2}$. An element $x^{*} \in D$ is called a fixed point of $T$ if $T x^{*}=x^{*}$. For details on cone theory, see $[16,17]$.

Given $e>\theta$, let

$\overline{P_{e}}=\{x \in E \mid$ there exist $l=l(x)>0$ such that $0 \leq x \leq l e\}$,

$$
\begin{aligned}
P_{e}=\left\{x \in E \mid \text { there exist } l_{1}=l_{1}(x)>0,\right. & \\
& \left.l_{2}=l_{2}(x)>0 \text { such that } l_{1} e \leq x \leq l_{2} e\right\} .
\end{aligned}
$$

Then $P_{e} \subset \overline{P_{e}} \subset P$ and

$$
\begin{aligned}
& \forall x, y \in P_{e}, \quad \exists 0<\mu_{0}<1<l_{0}<+\infty \\
& \text { such that } \mu_{0} y \leq x \leq l_{0} y .
\end{aligned}
$$

Lemma 1. Let $P$ be a normal cone in $E$ and $T: P \rightarrow P$ an increasing operator. Suppose that

(H1) $T\left(P_{e}\right) \subset P_{e}$;

(H2) for any $r \in(0,1)$ and $[y, z] \subset P_{e}$ there exists $\eta(r, y$, z) $>0$ such that

$$
T(r x) \geq r(1+\eta(r, y, z)) T x, \quad \forall x \in[y, z], r \in(0,1) .
$$

Then $T$ has a unique fixed point $x^{*}$ in $P_{e}$ if and only if there exist $u, v \in P_{e}$ such that $u \leq T u \leq T v \leq v$. Moreover, for any initial value $u_{0} \in P_{e}$ and a sequence $u_{n}=T u_{n-1}(n=1,2, \ldots)$, one has $\lim _{n \rightarrow+\infty}\left\|u_{n}-x^{*}\right\|=0$.

The proof of Lemma 1 is standard; we omit it here.

Definition 2 (see [14]). An operator $T: P \rightarrow P$ is said to be generalized $\alpha$-concave if it satisfies (H1) and the following condition:
(H3) there exists an $\alpha:(0,1) \rightarrow(0,1)$ such that

$$
T(r x) \geq r^{\alpha(r)} T x, \quad \forall x \in P_{e}, r \in(0,1) .
$$

From Lemma 1 it is easy to show that the following lemma holds.

Lemma 3 (see [15]). Let $P$ be a normal cone of $E$ and $T: P \rightarrow$ $P$ increasing generalized $\alpha$-concave. Then $T$ has a unique fixed point $x^{*}$ in $P_{e}$. Moreover, for any initial value $u_{0} \in P_{e}$ and $a$ sequence $u_{n}=T u_{n-1}(n=1,2, \ldots)$, one has $\lim _{n \rightarrow+\infty} \| u_{n}-$ $x^{*} \|=0$.

In what follows, we introduce definitions of pseudo subhomogeneous operator and pseudo generalized $\alpha$-concave operator.

Definition 4. An operator $T: P \rightarrow P$ is said to be pseudo subhomogeneous if it satisfies

(H4) $T\left(P_{e}\right) \subset \overline{P_{e}}$;

(H5) $T(r x) \geq r T x, \forall x \in P_{e}, r \in(0,1)$.

Definition 5. An operator $T: P \rightarrow P$ is said to be pseudo generalized $\alpha$-concave if it satisfies (H3) and (H4).

Remark 6. An increasing pseudo subhomogeneous operator and an increasing pseudo generalized $\alpha$-concave operator may have no fixed point in $P_{e}$. For example, Let $E=$ $C[0,1], P=\{x \in E \mid x(t) \geq 0, t \in[0,1]\}, e(t) \equiv 1$, and $T_{1} x(t)=(1-t) x(t) /(1+x(t))$. Obviously, $T_{1}: P \rightarrow P$ is increasing pseudo subhomogeneous, but $T_{1}$ has no fixed point in $P_{e}$. Let

$$
T_{2} x(t)=\int_{0}^{1} t(1-s) x^{\alpha}(s) d s, \quad x \in P,
$$

$0<\alpha<1$. Clearly, $T_{2}: P \rightarrow P$ is increasing pseudo generalized $\alpha$-concave. Since $T_{2} x(0)=0$ for $x \in P_{e}$, then $T_{2} x \notin P_{e}$ for all $x \in P_{e}$. Therefore, $T_{2}$ is not generalized $\alpha$ concave and $T_{2}$ has no fixed point in $P_{e}$.

Remark 7. From Definitions 4 and 5, it is clear that a pseudo generalized $\alpha$-concave operator is a pseudo subhomogeneous operator.

Remark 8. It is easy to show that (8) is equivalent to

$$
T(s x) \leq s^{\alpha(1 / s)} T x, \quad x \in P_{e}, s>1,
$$

and (H5) is equivalent to

$$
T(s x) \leq s T x, \quad x \in P_{e}, s>1 .
$$

\section{Positive Solutions of Operator Equation}

In this section, we assume that $E$ is a real Banach space, $P$ is a normal cone in $E$ with the normal constant $N, e>\theta$, and $A, B: P \rightarrow P$ are increasing operators. 
Theorem 9. Assume that $A$ is a generalized $\alpha$-concave operator and $B$ is a pseudo subhomogeneous operator. Then the following four results are true.

(i) There exists a $\lambda^{*}>0$ such that (1) has a unique solution $x_{\lambda}$ in $P_{e}$ for $\lambda \in\left[0, \lambda^{*}\right)$. For any initial value $u_{0} \in P_{e}$, set $u_{n}=L\left(\lambda, u_{n-1}\right)(n=1,2, \ldots)$; then $\lim _{n \rightarrow+\infty} \| u_{n}-$ $x_{\lambda} \|=0$.

(ii) Equation (1) has no solution in $P_{e}$ for $\lambda \geq \lambda^{*}$.

(iii) $x_{\lambda}$ is increasing in $\lambda$ for $\lambda \in\left[0, \lambda^{*}\right)$.

(iv) $x_{\lambda}$ is continuous with respect to $\lambda$ for $\lambda \in\left[0, \lambda^{*}\right)$.

Proof. By Lemma 3, $A$ has a unique fixed point $x_{0} \in P_{e}$ and

$$
L\left(\lambda, x_{0}\right)=A x_{0}+\lambda B x_{0} \geq A x_{0}=x_{0}, \quad \lambda \geq 0 .
$$

If $\left.B\right|_{P_{e}} \equiv \theta,\left.L(\lambda, \cdot)\right|_{P_{e}}=A$ for any $\lambda \geq 0$. Set $\lambda^{*}=+\infty$; it is obvious that conclusions (i)-(iv) hold.

If $\left.B\right|_{P_{e}} \neq \equiv \theta$, there exists $\bar{x} \in P_{e}$ such that $B \bar{x}>\theta$. By (6), for any $x \in P_{e}$, there exists $\mu_{0} \in(0,1)$ such that $\mu_{0} \bar{x} \leq x$. Moreover, Definition 4 shows that there exists $l_{0} \in(1,+\infty)$ such that

$$
\theta<\mu_{0} B \bar{x} \leq B x \leq l_{0} x_{0}, \quad x \in P_{e} .
$$

The facts that $A\left(P_{e}\right) \subset P_{e}$ and $B\left(P_{e}\right) \subset \overline{P_{e}}$ imply that

$$
L\left(\lambda, P_{e}\right) \subset P_{e}, \quad \lambda \geq 0 .
$$

Next, we prove all statements by five steps.

Step 1. Existence of the critical value $\lambda^{*}$. Set

$$
\begin{aligned}
\Lambda=\left\{\lambda \geq 0 \mid \text { there exists } y_{\lambda} \in P_{e}\right. \\
\left.\quad \text { such that } x_{0} \leq y_{\lambda}, L\left(\lambda, y_{\lambda}\right) \leq y_{\lambda}\right\},
\end{aligned}
$$

and $\lambda^{*}=\sup \Lambda$. Now we show that $\Lambda=\left[0, \lambda^{*}\right)$.

Define a mapping $\rho: P_{e} \rightarrow[0,+\infty)$ by

$$
\rho(x)=\inf \left\{\tau>0 \mid B x \leq \tau x_{0}\right\}, \quad x \in P_{e} .
$$

By (13) it is obvious that $0<\rho(x)<+\infty$. In addition, for any $x_{1}, x_{2} \in P_{e}, x_{1} \leq x_{2}$, we have $B x_{1} \leq B x_{2} \leq \rho\left(x_{2}\right) x_{0}$, which implies that $\rho\left(x_{1}\right) \leq \rho\left(x_{2}\right)$. That is, $\rho(x)$ is increasing in $x$ for $x \in P_{e}$.

For a given $s_{0}>1$, set $y_{0}=s_{0} x_{0}$. Then $y_{0} \in P_{e}$ and $x_{0} \leq$ $y_{0}$. It follows from Remark 8 that

$$
\begin{array}{r}
L\left(\lambda, y_{0}\right) \leq s_{0}\left(s_{0}^{\alpha\left(1 / s_{0}\right)-1}+\lambda \rho\left(x_{0}\right)\right) x_{0} \leq y_{0}, \\
\lambda \in\left[0, \frac{1}{\rho\left(x_{0}\right)}\left(1-s_{0}^{\alpha\left(1 / s_{0}\right)-1}\right)\right] .
\end{array}
$$

This means that

$$
\begin{aligned}
& {\left[0, \frac{1}{\rho\left(x_{0}\right)}\left(1-s_{0}^{\alpha\left(1 / s_{0}\right)-1}\right)\right] \subset \Lambda,} \\
& \lambda^{*} \geq \frac{1}{\rho\left(x_{0}\right)}\left(1-s_{0}^{\alpha\left(1 / s_{0}\right)-1}\right)>0 .
\end{aligned}
$$

We assert that $\lambda^{*} \notin \Lambda$. If $\lambda^{*}=+\infty$, from (15) it is obvious that $\lambda^{*} \notin \Lambda$. Suppose that $\lambda^{*}<+\infty$ and $\lambda^{*} \in \Lambda$. Then again by (15) there exists $y_{\lambda^{*}} \in P_{e}$ with $x_{0} \leq y_{\lambda^{*}}$ such that $L\left(\lambda^{*}, y_{\lambda^{*}}\right) \leq$ $y_{\lambda^{*}}$. Set $v_{0}=s_{1} y_{\lambda^{*}}$ for a given $s_{1}>1$. Then $v_{0} \in P_{e}$ and $x_{0} \leq v_{0}$. Note that $\left(1 / \rho\left(y_{\lambda^{*}}\right)\right)\left(1-s_{1}^{\alpha\left(1 / s_{1}\right)-1}\right)>0$; we can take a number $\delta>0$ sufficiently small such that

$$
\delta<\frac{1}{\rho\left(y_{\lambda^{*}}\right)}\left(1-s_{1}^{\alpha\left(1 / s_{1}\right)-1}\right) .
$$

From (16)-(19) and the fact that $x_{0}=A x_{0} \leq A y_{\lambda^{*}}$, we obtain

$$
\begin{aligned}
L\left(\lambda^{*}+\delta, v_{0}\right)= & A\left(s_{1} y_{\lambda^{*}}\right)+\left(\lambda^{*}+\delta\right) B\left(s_{1} y_{\lambda^{*}}\right) \\
\leq & s_{1}\left(A y_{\lambda^{*}}+\lambda^{*} B y_{\lambda^{*}}\right)+\left(s_{1}^{\alpha\left(1 / s_{1}\right)}-s_{1}\right) A y_{\lambda^{*}} \\
& +\delta s_{1} B y_{\lambda^{*}} \\
\leq & s_{1} L\left(\lambda^{*}, y_{\lambda^{*}}\right)-\left(s_{1}-s_{1}^{\alpha\left(1 / s_{1}\right)}\right) A x_{0} \\
& +\delta s_{1} \rho\left(y_{\lambda^{*}}\right) x_{0} \\
\leq & s_{1} y_{\lambda^{*}}+\left(\delta s_{1} \rho\left(y_{\lambda^{*}}\right)-\left(s_{1}-s_{1}^{\alpha\left(1 / s_{1}\right)}\right)\right) x_{0} \\
\leq & s_{1} y_{\lambda^{*}}=v_{0},
\end{aligned}
$$

which means that $\lambda^{*}+\delta \in \Lambda$. This contradicts the definition of $\lambda^{*}$. Therefore, we conclude that

$$
\Lambda=\left[0, \lambda^{*}\right) \text {. }
$$

Step 2. Conclusion (i) holds. For given $\lambda \in\left[0, \lambda^{*}\right)$, consider (1). Since $A$ and $B$ are increasing, $L(\lambda, x)$ is increasing in $x$. Moreover, combining (12) and (15) gives

$$
x_{0} \leq L\left(\lambda, x_{0}\right) \leq L\left(\lambda, y_{\lambda}\right) \leq y_{\lambda} .
$$

Besides, by (H2) and (H4) we obtain

$$
\begin{aligned}
& L(\lambda, r x) \geq r^{\alpha(r)} A x+\lambda r B x \\
& \geq r L(\lambda, x)+\left(r^{\alpha(r)}-r\right) A x \\
& \geq r\left(1+\frac{r^{\alpha(r)}-r}{r} \varphi(x, \lambda)\right) L(\lambda, x), \\
& x \in P_{e}, r \in(0,1),
\end{aligned}
$$

where

$$
\varphi(\lambda, x)=\sup \{\tau>0 \mid A x \geq \tau L(\lambda, x)\}, \quad x \in P_{e} .
$$

For any $u, v \in P_{e}$ with $u \leq v$, (H1) implies that $A u, A v \in P_{e}$. Further, there exists $0<\epsilon \leq 1$ such that $\epsilon A v \leq A u$. Hence,

$$
\begin{aligned}
A x & \geq A u \geq \epsilon A v \geq \epsilon \varphi(\lambda, v) L(\lambda, v) \\
& \geq \epsilon \varphi(\lambda, v) L(\lambda, x), \quad x \in[u, v] .
\end{aligned}
$$


Evidently,

$$
\varphi(\lambda, x) \geq \epsilon \varphi(\lambda, v)>0, \quad \forall x \in[u, v]
$$

Therefore, let $\eta(r, u, v)=\left(\epsilon\left(r^{\alpha(r)}-r\right) / r\right) \varphi(\lambda, v)$; then it follows from (23) and (26) that

$$
L(\lambda, r x) \geq r(1+\eta(r, u, v)) L(\lambda, x), \quad x \in[u, v], r \in(0,1) .
$$

The application of Lemma 1 concludes the proof of (i).

Step 3. Conclusion (ii) holds. Suppose that there exists $\lambda^{\prime} \geq$ $\lambda^{*}$ such that $L\left(\lambda^{\prime}, x\right)=x$ has a solution $x_{\lambda^{\prime}}$ in $P_{e}$. Since $x_{0}, x_{\lambda^{\prime}} \in P_{e}$, by (6) there exists $s^{\prime}>1$ such that $x_{0} \leq s^{\prime} x_{\lambda^{\prime}}$. Set $y_{\lambda^{\prime}}=s^{\prime} x_{\lambda^{\prime}}$. Then

$$
\begin{aligned}
L\left(\lambda^{\prime}, y_{\lambda^{\prime}}\right) & =A\left(s^{\prime} x_{\lambda^{\prime}}\right)+\lambda^{\prime} B\left(s^{\prime} x_{\lambda^{\prime}}\right) \\
& \leq s^{\prime}\left(\left(s^{\prime}\right)^{\alpha\left(1 / s^{\prime}\right)-1} A x_{\lambda^{\prime}}+\lambda^{\prime} B x_{\lambda^{\prime}}\right) \leq s^{\prime} x_{\lambda^{\prime}}=y_{\lambda^{\prime}},
\end{aligned}
$$

which means that $\lambda^{\prime} \in \Lambda$. Equation (21) implies that $\lambda^{\prime}<\lambda^{*}$, which is a contradiction to the hypothesis $\lambda^{\prime} \geq \lambda^{*}$.

Step 4. Conclusion (iii) holds. Let $\lambda_{1}, \lambda_{2} \in\left[0, \lambda^{*}\right)$ with $\lambda_{1} \leq$ $\lambda_{2}$. Then $L\left(\lambda_{1}, x_{\lambda_{2}}\right) \leq L\left(\lambda_{2}, x_{\lambda_{2}}\right)=x_{\lambda_{2}}$, and, further, $x_{0} \leq$ $L\left(\lambda_{1}, x_{0}\right) \leq L\left(\lambda_{1}, x_{\lambda_{2}}\right) \leq x_{\lambda_{2}}$. By the proof of conclusion (i), $x=L\left(\lambda_{1}, x\right)$ has a unique solution $x_{\lambda_{1}}^{*} \in\left[x_{0}, x_{\lambda_{2}}\right]$ in $P_{e}$, which implies that $x_{\lambda_{1}}=x_{\lambda_{1}}^{*}$. Thus, $x_{\lambda_{1}} \leq x_{\lambda_{2}}$.

Step 5. Conclusion (iv) holds. Let $\lambda_{0} \in\left(0, \lambda^{*}\right)$. By conclusion (iii) we have

$$
x_{\lambda} \leq x_{\lambda_{0}}, \quad \forall 0<\lambda<\lambda_{0}
$$

Let

$$
\tau_{\lambda}=\sup \left\{\tau>0 x_{\lambda} \geq \tau x_{\lambda_{0}}\right\}, \quad 0<\lambda<\lambda_{0}
$$

Then $\tau_{\lambda}$ is nondecreasing in $\lambda$ for $\lambda \in\left[0, \lambda_{0}\right)$, and

$$
0<\tau_{\lambda} \leq 1, \quad x_{\lambda} \geq \tau_{\lambda} x_{\lambda_{0}}, \quad 0<\lambda<\lambda_{0}
$$

We assert that

$$
\lim _{\lambda \rightarrow \lambda_{0}^{-}} \tau_{\lambda}=1
$$

if, otherwise, there exists a sequence $\left\{\lambda_{n}\right\}$

$$
0<\lambda_{1} \leq \lambda_{2} \leq \cdots \leq \lambda_{n} \leq \cdots \leq \lambda_{0}, \quad \lim _{n \rightarrow+\infty} \lambda_{n}=\lambda_{0}
$$

such that

$$
\lim _{n \rightarrow+\infty} \tau_{\lambda_{n}}=\tau_{0}, \quad \tau_{\lambda_{n}} \leq \tau_{0}<1
$$

By (23), (24), and (30), we obtain

$$
\begin{aligned}
x_{\lambda_{n}} & =L\left(\lambda_{n}, x_{\lambda_{n}}\right) \geq L\left(\lambda_{n}, \tau_{\lambda_{n}} x_{\lambda_{0}}\right) \\
& =L\left(\lambda_{n}, \frac{\tau_{\lambda_{n}}}{\tau_{0}} \tau_{0} x_{\lambda_{0}}\right) \geq \frac{\tau_{\lambda_{n}}}{\tau_{0}} L\left(\lambda_{n}, \tau_{0} x_{\lambda_{0}}\right) \\
& \geq \frac{\lambda_{n}}{\lambda_{0}} \frac{\tau_{\lambda_{n}}}{\tau_{0}} L\left(\lambda_{0}, \tau_{0} x_{\lambda_{0}}\right) \\
& \geq \frac{\lambda_{n}}{\lambda_{0}} \tau_{\lambda_{n}}\left(1+\frac{\tau_{0}^{\alpha\left(\tau_{0}\right)}-\tau_{0}}{\tau_{0}} \varphi\left(x_{\lambda_{0}}, \lambda_{0}\right)\right) x_{\lambda_{0}} .
\end{aligned}
$$

Therefore,

$$
\tau_{\lambda_{n}} \geq \frac{\lambda_{n}}{\lambda_{0}} \tau_{\lambda_{n}}\left(1+\frac{\tau_{0}^{\alpha\left(\tau_{0}\right)}-\tau_{0}}{\tau_{0}} \varphi\left(x_{\lambda_{0}}, \lambda_{0}\right)\right) .
$$

That is,

$$
\frac{\lambda_{n}}{\lambda_{0}}\left(1+\frac{\tau_{0}^{\alpha\left(\tau_{0}\right)}-\tau_{0}}{\tau_{0}} \varphi\left(x_{\lambda_{0}}, \lambda_{0}\right)\right) \leq 1
$$

Taking the limit $n \rightarrow+\infty$, we get

$$
1+\frac{\tau_{0}^{\alpha\left(\tau_{0}\right)}-\tau_{0}}{\tau_{0}} \varphi\left(x_{\lambda_{0}}, \lambda_{0}\right) \leq 1,
$$

which is a contradiction. So, (32) holds.

From (29), (31), and (32), we have

$$
\left\|x_{\lambda_{0}}-x_{\lambda}\right\| \leq N\left(1-\tau_{\lambda}\right)\left\|x_{\lambda_{0}}\right\|, \quad 0<\lambda<\lambda_{0},
$$

which implies that $\left\|x_{\lambda_{0}}-x_{\lambda}\right\| \rightarrow 0$ as $\lambda \rightarrow \lambda_{0}^{-}$.

A similar argument shows that, for any $\lambda_{0} \in\left[0, \lambda^{*}\right), \| x_{\lambda}-$ $x_{\lambda_{0}} \| \rightarrow 0$ as $\lambda \rightarrow \lambda_{0}^{+}$. Thus, conclusion (iv) holds. The proof of Theorem 9 is complete. result.

Noting (18) and (21), we can easily obtain the following

Theorem 10. Assume that the hypotheses in Theorem 9 hold. If $\lim _{r \rightarrow 0^{+}} r^{1-\alpha(r)}=0$, then $\lambda^{*}$ in Theorem 9 satisfies

$$
\lambda^{*} \geq \frac{1}{\rho\left(x_{0}\right)}
$$

where $\rho\left(x_{0}\right)$ are defined by (16).

Corollary 11. Assume that $A$ is a generalized $\alpha_{1}$-concave operator and $B$ is a pseudo generalized $\alpha_{2}$-concave operator. Then

(i) (1) has a unique solution $x_{\lambda} \in P_{e}$ for $\lambda \in[0,+\infty)$. Moreover, for any $u_{0} \in P_{e}$, set $u_{n}=L\left(\lambda, u_{n-1}\right)(n=$ $1,2, \ldots)$; then $\lim _{n \rightarrow+\infty}\left\|u_{n}-x_{\lambda}\right\|=0$;

(ii) $x_{\lambda}$ is increasing in $\lambda$ for $\lambda \in[0,+\infty)$;

(iii) $x_{\lambda}$ is continuous with respect to $\lambda$ for $\lambda \in[0,+\infty)$; 
(iv) either $x_{\lambda}=x_{0}$ (the unique fixed point of $A$ in $P_{e}$ ) or $\lim _{\lambda \rightarrow+\infty}\left\|x_{\lambda}\right\|=+\infty$.

Proof. From Definitions 2 and 4, it is clear that $L\left(\lambda, P_{e}\right) \subset P_{e}$. Since $A$ and $B$ are increasing, $L(\lambda, x)$ is increasing in $x$ for any $\lambda \geq 0$. Let $\alpha(r)=\max \left\{\alpha_{1}(r), \alpha_{2}(r)\right\}$; then

$$
\begin{aligned}
L(\lambda, r x) & =A(r x)+\lambda B(r x) \\
& \geq r^{\alpha_{1}(r)} A x+\lambda r^{\alpha_{2}(r)} B x \\
& \geq r^{\alpha(r)} L(\lambda, x), \quad x \in P_{e}, r \in(0,1) .
\end{aligned}
$$

Thus, conclusion (i) follows from Lemma 3. Similar to the proofs of Theorem 9, the proofs of (ii) and (iii) can be completed.

Note that

$$
\begin{gathered}
x_{\lambda}=A x_{\lambda}=x_{0}, \quad \text { if }\left.B\right|_{P_{e}} \equiv \theta, \\
x_{\lambda}=A x_{\lambda}+\lambda B x_{\lambda} \geq \lambda B x_{\lambda} \geq \lambda B x_{0}, \quad \text { if }\left.B\right|_{P_{e}} \not \equiv \theta,
\end{gathered}
$$

and, therefore, normality of $P$ implies that conclusion (iv) holds. This ends the proof.

Theorem 12. Assume that $A$ is a pseudo generalized $\alpha_{1}$ concave operator and $B$ is a generalized $\alpha_{2}$-concave operator. Then,

(i) (1) has a unique solution $x_{\lambda} \in P_{e}$ for $\lambda \in(0,+\infty)$. Moreover, for any $u_{0} \in P_{e}$, set $u_{n}=L\left(\lambda, u_{n-1}\right)(n=$ $1,2, \ldots)$; then $\lim _{n \rightarrow+\infty}\left\|u_{n}-x_{\lambda}\right\|=0$;

(ii) $x_{\lambda}$ is increasing with respect to $\lambda$ for $\lambda \in(0,+\infty)$;

(iii) $x_{\lambda}$ is continuous with respect to $\lambda$ for $\lambda \in(0,+\infty)$;

(iv) $\lim _{\lambda \rightarrow 0}\left\|x_{\lambda}-A x_{\lambda}\right\|=0 ; \lim _{\lambda \rightarrow+\infty}\left\|x_{\lambda}\right\|=+\infty$.

Proof. Similar to the proof of Corollary 11, the proofs of (i), (ii), and (iii) can be completed. To prove (iv), let $x_{1} \in P_{e}$ be the unique solution of (1) with $\lambda=1$. From conclusion (ii) of this theorem, we obtain

$$
\begin{gathered}
\theta<x_{\lambda}-A x_{\lambda}=\lambda B x_{\lambda} \leq \lambda B x_{1}, \quad 0<\lambda<1, \\
x_{\lambda}=A x_{\lambda}+\lambda B x_{\lambda} \geq \lambda B x_{\lambda} \geq \lambda B x_{1}>\theta, \quad \lambda>1 .
\end{gathered}
$$

Therefore,

$$
\begin{gathered}
\left\|x_{\lambda}-A x_{\lambda}\right\| \leq N \lambda\left\|B x_{1}\right\|, \quad 0<\lambda<1, \\
\left\|x_{\lambda}\right\| \geq \frac{\lambda}{N}\left\|B x_{1}\right\|, \quad \lambda>1,
\end{gathered}
$$

which implies that $\lim _{\lambda \rightarrow 0}\left\|x_{\lambda}-A x_{\lambda}\right\|=0$ and $\lim _{\lambda \rightarrow+\infty}\left\|x_{\lambda}\right\|$ $=+\infty$. This ends the proof.

Next, we discuss the case of (1) with $L(\lambda, x)=x_{0}+$ $\lambda B x, x_{0} \in E$; that is,

$$
x_{0}+\lambda B x=x
$$

which can be widely applied to various problems for differential equations.
Theorem 13. Assume that $x_{0} \in P_{e}$ and $B$ is a pseudo subhomogeneous operator. Then

(i) there exists a $\lambda^{*} \geq\left(1 / \rho_{0}\right)>0$ such that (45) has a unique solution $x_{\lambda} \in P_{e}$ for $\lambda \in\left[0, \lambda^{*}\right)$. Moreover, for any $u_{0} \in P_{e}$ and a sequence $u_{n}=x_{0}+\lambda B u_{n-1}(n=$ $1,2, \ldots)$, one has $\lim _{n \rightarrow+\infty}\left\|u_{n}-x_{\lambda}\right\|=0$;

(ii) (45) has no solution in $P_{e}$ for $\lambda \geq \lambda^{*}$;

(iii) $x_{\lambda}$ is increasing in $\lambda$ for $\lambda \in\left[0, \lambda^{*}\right)$;

(iv) $x_{\lambda}$ is continuous with respect to $\lambda$ for $\lambda \in\left[0, \lambda^{*}\right)$; moreover, $\lim _{\lambda \rightarrow 0}\left\|x_{\lambda}-x_{0}\right\|=0$;

(v) further, if $B x \in \overline{P_{e}}$ for $x \geq x_{0}$ and $B$ is completely continuous, then $\lim _{\lambda \rightarrow \lambda^{*}-0}\left\|x_{\lambda}\right\|=+\infty$.

Here $\rho_{0}=\inf \left\{\tau>0 \mid B x_{0} \leq \tau x_{0}\right\}$.

Proof. Define an operator $A$ by $A x=x_{0}$ for $x \in P$. Then $A: P \rightarrow P_{e}$ is increasing and $x_{0}$ is the unique fixed point of $A$ in $P_{e}$.

Conclusions (i)-(iv) can be proved similarly to the proof of Theorem 9. We only prove conclusion (v) by considering the following two cases.

Case $1\left(\lambda^{*}=+\infty\right)$. Note that $x_{\lambda}=x_{0}+\lambda B x_{\lambda} \geq \lambda B x_{\lambda} \geq \lambda B x_{0}$ for $\lambda \in[0,+\infty)$; it is clear that $\lim _{\lambda \rightarrow \lambda^{*}-0}\left\|x_{\lambda}\right\|=+\infty$.

Case $2\left(\lambda^{*}<+\infty\right)$. In this case, suppose, to the contrary, that $\lim _{\lambda \rightarrow \lambda^{*}-0}\left\|x_{\lambda}\right\| \neq+\infty$. Then, there exists a nondecreasing sequence $\left\{\lambda_{n}\right\} \subset\left[0, \lambda^{*}\right)$ and a constant $M>0$ such that $\lim _{n \rightarrow+\infty} \lambda_{n}=\lambda^{*}$ and $\left\|x_{\lambda_{n}}\right\| \leq M$.

Since $B$ is completely continuous, there exist $x^{*} \in P$ and subsequence $\left\{\lambda_{n_{i}}\right\} \subset\left\{\lambda_{n}\right\}$ such that $\lim _{i \rightarrow+\infty} B x_{\lambda_{n_{i}}}=x^{*}$. Taking the limitation $i \rightarrow+\infty$ to both sides of

$$
x_{\lambda_{n_{i}}}=x_{0}+\lambda_{n_{i}} B x_{\lambda_{n_{i}}},
$$

we have $\lim _{i \rightarrow+\infty} x_{\lambda_{n_{i}}}=x_{0}+\lambda^{*} x^{*}$. Equation (46) implies that

$$
x_{0}+\lambda^{*} x^{*}=x_{0}+\lambda^{*} B\left(x_{0}+\lambda^{*} x^{*}\right) .
$$

The relation $x_{0}+\lambda^{*} x^{*}>x_{0}$ shows that $B\left(x_{0}+\lambda^{*} x^{*}\right) \in \overline{P_{e}}$. Further, $x_{0}+\lambda^{*} B\left(x_{0}+\lambda^{*} x^{*}\right) \in P_{e}$. This means that $x_{0}+\lambda^{*} x^{*} \epsilon$ $P_{e}$. Therefore, (47) gives rise to the contradiction $\lambda^{*} \in\left[0, \lambda^{*}\right)$. This finishes the proof.

Corollary 14. Assume that $x_{0} \in P_{e}$ and $B$ is pseudo generalized $\alpha$-concave. Then,

(i) (45) has a unique solution $x_{\lambda} \in P_{e}$ for $\lambda \in[0,+\infty)$. Moreover, for any $u_{0} \in P_{e}$, set $u_{n}=x_{0}+\lambda B u_{n-1} \quad(n=$ $1,2, \ldots)$; then $\lim _{n \rightarrow+\infty}\left\|u_{n}-x_{\lambda}\right\|=0$;

(ii) $x_{\lambda}$ is increasing with respect to $\lambda$ for $\lambda \in[0,+\infty)$;

(iii) $x_{\lambda}$ is continuous with respect to $\lambda$ for $\lambda \in[0,+\infty)$;

(iv) either $x_{\lambda}=x_{0}, \lambda \geq 0$ or $\lim _{\lambda \rightarrow+\infty}\left\|x_{\lambda}\right\|=+\infty$. 
Proof. It is obvious that $L(\lambda, x)=x_{0}+\lambda B x$ is increasing in $x$ and $L\left(\lambda, P_{e}\right) \subset P_{e}$ for any $\lambda \geq 0$. In addition,

$$
\begin{aligned}
L(\lambda, r x)= & x_{0}+\lambda B(r x) \geq x_{0} \\
& +\lambda r^{\alpha(r)} B x \geq r^{\alpha(r)} L(\lambda, x), \quad x \in P_{e}, r \in(0,1) .
\end{aligned}
$$

This means that $L(\lambda, \cdot)$ is generalized $\alpha$-concave. Thus, conclusion (i) follows from Lemma 3. Similarly to the proof of Corollary 11, the proofs of (ii), (iii), and (iv) can be completed.

From the proofs of Theorem 12, Theorem 13, and Corollary 14 , we easily prove the following results.

Theorem 15. Assume that $x_{0} \in \overline{P_{e}}$ and $B$ is a generalized $\alpha$ concave operator. Then,

(i) (45) has a unique solution $x_{\lambda} \in P_{e}$ for $\lambda \in(0,+\infty)$. Moreover, for any $u_{0} \in P_{e}$, set $u_{n}=x_{0}+\lambda B u_{n-1}(n=$ $1,2, \ldots)$; then $\lim _{n \rightarrow+\infty}\left\|u_{n}-x_{\lambda}\right\|=0$;

(ii) $x_{\lambda}$ is increasing in $\lambda$ and $x_{\lambda} \geq x_{0}$ for $\lambda \in(0,+\infty)$;

(iii) $x_{\lambda}$ is continuous with respect to $\lambda$ for $\lambda \in(0,+\infty)$;

(iv) $\lim _{\lambda \rightarrow 0}\left\|x_{\lambda}-x_{0}\right\|=0$ and $\lim _{\lambda \rightarrow+\infty}\left\|x_{\lambda}\right\|=+\infty$.

Remark 16. Different from Theorem 9 and Corollary 11, even if (1) satisfies the conditions in Theorem 12, (1) may not have a fixed point in $P_{e}$ when $\lambda=0$.

\section{Three-Point Nonlinear Boundary Value Problem}

In this section, based on the discussion of the previous section, we study the existence and uniqueness of positive solutions for the three-point $\operatorname{BVP}(2)$ and the dependence of solutions on the parameter $\lambda$.

In what follows, set $E=C[0,1]$, the Banach space of continuous functions on $[0,1]$ with the norm $\|x\|=$ $\max _{t \in[0,1]}|x(t)|$. Consider that $P=\{x \in C[0,1] \mid x(t) \geq 0, t \in$ $[0,1]\}$. It is clear that $P$ is a normal cone with the normality constant 1; $P_{e}$ is given as in (5) with $e(t)=\tau+t$. Let

$G(t, s)$

$$
\begin{aligned}
= & \frac{1}{1+\tau-\sigma(\xi+\tau)} \\
& \times \begin{cases}(1-\sigma \xi-(1-\sigma) t)(\tau+s), & 0 \leq s \leq \min \{\xi, t\}, \\
(1-t)(\tau+s)+\sigma(\xi+\tau)(t-s), & \xi \leq s \leq t \leq 1, \\
(\tau+t)(1-s-\sigma(\xi-s)), & 0 \leq t \leq s \leq \xi, \\
(\tau+t)(1-s), & \max \{\xi, t\} \leq s \leq 1 .\end{cases}
\end{aligned}
$$

It is easy to prove that

$$
\begin{aligned}
0 & \leq G(t, s) \\
& \leq \frac{1+\sigma}{1+\tau-\sigma(\xi+\tau)}(\tau+t)(1-s), \quad t, s \in[0,1] .
\end{aligned}
$$

Define two operators $A: P \rightarrow C[0,1]$ and $B: P \rightarrow C[0,1]$ by

$$
\begin{gathered}
A x(t)=\frac{\tau+t}{1+\tau-\sigma(\xi+\tau)} \int_{0}^{1} h(x(s)) d s \\
\quad+\int_{0}^{1} G(t, s) g(s) d s . \\
B x(t)=\int_{0}^{1} G(t, s) f(s, x(s)) d s .
\end{gathered}
$$

It is clear that a positive solution of $\mathrm{BVP}(2)$ is equivalent to nontrivial solution of (1) in $P$.

The following lemma can be proved easily by the AscoliArzela theorem.

Lemma 17. $B: P \rightarrow C[0,1]$ is completely continuous.

The following hypotheses are needed in this section.

(L1) $f:[0,1] \times[0,+\infty) \rightarrow[0,+\infty)$ is continuous.

(L2) $f(t, x)$ is increasing in $x \in[0,+\infty)$ for fixed $t \in[0,1]$ and

$$
\begin{array}{r}
f(t, r x) \geq r f(t, x), \quad \forall t \in[0,1], \\
r \in(0,1), \quad x \in[0,+\infty) .
\end{array}
$$

(L3) $h:[0,+\infty) \rightarrow[0,+\infty)$ is continuous and $h(x) \not \equiv 0$.

(L4) $h(x)$ is increasing in $x \in[0,+\infty)$ and there exists a function $\alpha(r) \in(0,1)$ such that

$$
h(r x) \geq r^{\alpha(r)} h(x), \quad \forall r \in(0,1), \quad x \in(0,+\infty) .
$$

(L5) $g:[0,1] \rightarrow[0,+\infty)$ is continuous.

(L6) $\int_{0}^{1}(\tau+s)(1-s) g(s) d s>0$.

Lemma 18. Suppose that (L1) and (L2) hold. Then $B: P \rightarrow P$ is increasing and satisfies

(i) $B(P \backslash\{\theta\}) \subset \overline{P_{e}}$;

(ii) $B(r x) \geq r B x, x \in P_{e}, r \in(0,1)$.

Proof. From (50), (52), and (L1), it is clear that $B(P) \subset P$. By (L2) we obtain that $B$ is an increasing operator and, for any $x \in P \backslash\{\theta\}$,

$$
\begin{aligned}
B x(t) & =\int_{0}^{1} G(t, s) f(s, x(s)) d s \\
& \leq \frac{(1+\sigma) \int_{0}^{1}(1-s) f(s, x(s)) d s}{1+\tau-\sigma(\xi+\tau)}(\tau+t) \\
& \leq \frac{(1+\sigma) \int_{0}^{1}(1-s) f(s,\|x\|) d s}{1+\tau-\sigma(\xi+\tau)} e(t) \\
& \leq l(x) e(t),
\end{aligned}
$$


where $l(x) \geq \max \left\{\left((1+\sigma) \int_{0}^{1}(1-s) f(s,\|x\|) d s\right) /(1+\tau-\sigma(\xi+\right.$ $\tau)), 1\}$, and

$$
\begin{aligned}
B(r x)(t) & =\int_{0}^{1} G(t, s) f(s, r x(s)) d s \\
& \geq r \int_{0}^{1} G(t, s) f(s, x(s)) d s=r B x(t), \quad r \in(0,1) .
\end{aligned}
$$

The proof is complete.

Theorem 19. Suppose that (L1)-(L5) hold. Then there exists $\lambda^{*}>0$ such that $B V P(2)$ has a unique positive solution $x_{\lambda}(t)$ in $P_{e}$ for $\lambda \in\left[0, \lambda^{*}\right)$ and has no solution in $P_{e}$ for $\lambda \geq$ $\lambda^{*}$. Furthermore such a solution $x_{\lambda}(t)$ satisfies the following properties:

(i) for any $u_{0} \in P_{e}$, set

$$
\begin{aligned}
u_{n}(t)= & \frac{\tau+t}{1+\tau-\sigma(\xi+\tau)} \int_{0}^{1} h\left(u_{n-1}(s)\right) d s \\
& +\int_{0}^{1} G(t, s) g(s) d s \\
& +\lambda \int_{0}^{1} G(t, s) f\left(s, u_{n-1}(s)\right) d s,
\end{aligned}
$$

and then $u_{n}(t)$ uniformly converges to $x_{\lambda}(t)$ on $[0,1]$;

(ii) $x_{\lambda}(t)$ is nondecreasing in $\lambda$ for $\lambda \in\left[0, \lambda^{*}\right)$;

(iii) $x_{\lambda}(t)$ is continuous with respect to $\lambda$ for $\lambda \in\left[0, \lambda^{*}\right)$.

Proof. Consider $A$ and $B$ defined by (51) and (52). By (L3)(L5), $A: P \rightarrow P$ is increasing. For any $x \in P_{e}$, from (50) we have

$$
\begin{aligned}
& \frac{\int_{0}^{1} h(x(s)) d s}{1+\tau-\sigma(\xi+\tau)}(\tau+t) \\
& \quad \leq A x(t) \\
& \quad \leq \frac{\int_{0}^{1} h(x(s)) d s+(1+\sigma) \int_{0}^{1}(1-s) g(s) d s}{1+\tau-\sigma(\xi+\tau)}(\tau+t),
\end{aligned}
$$

which means that $A\left(P_{e}\right) \subset P_{e}$. In addition, for any $r \in(0,1)$ and $x \in P_{e}$, by (L4) we obtain

$$
\begin{aligned}
A(r x)(t)= & \frac{\tau+t}{1+\tau-\sigma(\xi+\tau)} \\
& \times \int_{0}^{1} h(r x(s)) d s+\int_{0}^{1} G(t, s) g(s) d s \\
\geq & r^{\alpha(r)}\left(\frac{\tau+t}{1+\tau-\sigma(\xi+\tau)} \int_{0}^{1} h(x(s)) d s\right. \\
& \left.+r^{-\alpha(r)} \int_{0}^{1} G(t, s) g(s) d s\right) \\
\geq & r^{\alpha(r)} A x(t) .
\end{aligned}
$$

So, the operator $A$ is generalized $\alpha$-concave. Lemma 18 implies that $B$ is pseudo subhomogeneous. The conclusion follows from Theorem 9 . The proof is complete.

In the following, we consider three special cases of $\operatorname{BVP}(2)$.

Case 1. $\operatorname{BVP}(2)$ has no perturbation; that is, $g(t) \equiv 0$.

From the proof of Theorem 19, we have the following result.

Theorem 20. Suppose that (L1)-(L4) hold. Then there exists $\lambda^{*}>0$ such that

$$
\begin{gathered}
-x^{\prime \prime}(t)=\lambda f(t, x(t)), \quad t \in(0,1), \\
x(0)=\tau x^{\prime}(0), \quad x(1)-\sigma x(\xi)=\int_{0}^{1} h(x(s)) d s
\end{gathered}
$$

have a unique positive solution $x_{\lambda}(t) \in P_{e}$ for $\lambda \in\left[0, \lambda^{*}\right)$ and no solution in $P_{e}$ for $\lambda \geq \lambda^{*}$. Furthermore such a solution $x_{\lambda}(t)$ satisfies the following properties:

(i) for any $u_{0} \in P_{e}$, set

$$
\begin{aligned}
u_{n}(t)= & \frac{\tau+t}{1+\tau-\sigma(\xi+\tau)} \int_{0}^{1} h\left(u_{n-1}(s)\right) d s \\
& +\lambda \int_{0}^{1} G(t, s) f\left(s, u_{n-1}(s)\right) d s,
\end{aligned}
$$

and then $u_{n}(t)$ uniformly converges to $x_{\lambda}(t)$ on $[0,1]$;

(ii) $x_{\lambda}(t)$ is nondecreasing in $\lambda$ for $\lambda \in\left[0, \lambda^{*}\right)$;

(iii) $x_{\lambda}(t)$ is continuous with respect to $\lambda$ for $\lambda \in\left[0, \lambda^{*}\right)$.

Case 2. The nonlinear boundary value control function $h(x)$ in $\operatorname{BVP}(2)$ reduces to the linear one $h_{0}(>0)$.

Theorem 21. Suppose that (L1), (L2), and (L5) hold. Then, there exists $\lambda^{*}>0$ such that

$$
\begin{gathered}
-x^{\prime \prime}(t)=\lambda f(t, x(t))+g(t), \quad t \in(0,1), \\
x(0)=\tau x^{\prime}(0), \quad x(1)-\sigma x(\xi)=h_{0}
\end{gathered}
$$

have a unique positive solution $x_{\lambda}(t) \in P_{e}$ for $\lambda \in\left[0, \lambda^{*}\right)$ and no solution in $P_{e}$ for $\lambda \geq \lambda^{*}$; furthermore such a solution $x_{\lambda}(t)$ satisfies the following properties:

(i) for any $u_{0} \in P_{e}$, set

$$
\begin{aligned}
u_{n}(t)= & \frac{\tau+t}{1+\tau-\sigma(\xi+\tau)} h_{0}+\int_{0}^{1} G(t, s) g(s) d s \\
& +\lambda \int_{0}^{1} G(t, s) f\left(s, u_{n-1}(s)\right) d s,
\end{aligned}
$$

and then $u_{n}(t)$ uniformly converges to $x_{\lambda}(t)$ on $[0,1]$;

(ii) $x_{\lambda}(t)$ is nondecreasing in $\lambda$ for $\lambda \in\left[0, \lambda^{*}\right)$;

(iii) $x_{\lambda}(t)$ is continuous with respect to $\lambda$ for $\lambda \in\left[0, \lambda^{*}\right)$;

(iv) $\lim _{\lambda \rightarrow \lambda^{*}-0}\left\|x_{\lambda}\right\|=+\infty$. 
Proof. Define operator $B$ as (52) and let

$$
x_{0}(t)=\frac{\tau+t}{1+\tau-\sigma(\xi+\tau)} h_{0}+\int_{0}^{1} G(t, s) g(s) d s .
$$

Then $x_{0} \in P_{e}$. By Lemmas 17 and 18, $B: P \rightarrow P$ is completely continuous and $B$ is pseudo subhomogeneous, and $B x \in \overline{P_{e}}$ for $x \geq x_{0}$. The application of Theorem 13 completes the proof.

Case 3. The nonlinear boundary value control function $h(x)$ in $\operatorname{BVP}(2)$ vanishes; that is, $h(x) \equiv 0$ for $t \in[0,1]$.

Theorem 22. Suppose that (L1), (L2), (L5), and (L6) hold. Then there exists $\lambda^{*}>0$ such that

$$
\begin{gathered}
-x^{\prime \prime}(t)=\lambda f(t, x(t))+g(t), \quad t \in(0,1), \\
x(0)=\tau x^{\prime}(0), \quad x(1)-\sigma x(\xi)=0
\end{gathered}
$$

have a unique positive solution $x_{\lambda}(t) \in P_{e}$ for $\lambda \in\left[0, \lambda^{*}\right)$ and no solution in $P_{e}$ for $\lambda \geq \lambda^{*}$; furthermore such a solution $x_{\lambda}(t)$ satisfies the following properties:

(i) for any $u_{0} \in P_{e}$, set

$$
u_{n}(t)=\int_{0}^{1} G(t, s) g(s) d s+\lambda \int_{0}^{1} G(t, s) f\left(s, u_{n-1}(s)\right) d s
$$

and then $u_{n}(t)$ uniformly converges to $x_{\lambda}(t)$ on $[0,1]$;

(ii) $x_{\lambda}(t)$ is nondecreasing in $\lambda$ for $\lambda \in\left[0, \lambda^{*}\right)$;

(iii) $x_{\lambda}(t)$ is continuous with respect to $\lambda$ for $\lambda \in\left[0, \lambda^{*}\right)$;

(iv) $\lim _{\lambda \rightarrow \lambda^{*}-0}\left\|x_{\lambda}\right\|=+\infty$.

Proof. Define operator $B$ as (52) and let

$$
x_{0}(t)=\int_{0}^{1} G(t, s) g(s) d s
$$

Note the monotonicity of $G(t, s)$ in $t$; it follows that

$$
\begin{aligned}
G(t, s) \geq & \frac{\sigma(1-\xi)(\tau+\xi)}{(1+\tau-\sigma(\xi+\tau))(\tau+1)^{2}} \\
& \times(\tau+s)(1-s)(\tau+t), \quad t, s \in[0,1]
\end{aligned}
$$

which together with (50), (L5), and (L6) leads to $x_{0} \in P_{e}$. By Lemma 18, $B: P \rightarrow P$ is pseudo subhomogeneous and $B x \in \overline{P_{e}}$ for $x \geq x_{0}$. The application of Theorem 13 finishes the proof.

\section{Conflict of Interests}

The authors declare that there is no conflict of interests regarding the publication of this paper.

\section{Acknowledgments}

Sincere thanks are due to the referees for their comments and suggestions. This research was supported by the NNSF of China (11361047, 10961020), the Science Foundation of Qinghai Province of China (2012-Z-910), Research Project Supported by Shanxi Scholarship Council of China (2013102), and the University Natural Science Research Development Foundation of Shanxi Province of China (20111021, 2013156).

\section{References}

[1] N. I. Ionkin, "The solution of a certain boundary value problem of the theory of heat conduction with a nonclassical boundary condition," Differential Equations, vol. 13, no. 2, pp. 294-304, 1977.

[2] C. P. Gupta, "Solvability of a three-point nonlinear boundary value problem for a second order ordinary differential equation," Journal of Mathematical Analysis and Applications, vol. 168, no. 2, pp. 540-551, 1992.

[3] Q. Yao, "Successive iteration and positive solution for nonlinear second-order three-point boundary value problems," Computers \& Mathematics with Applications, vol. 50, no. 3-4, pp. 433444, 2005.

[4] B. Liu, L. Liu, and Y. Wu, "Positive solutions for singular second order three-point boundary value problems," Nonlinear Analysis: Theory, Methods \& Applications, vol. 66, no. 12, pp. 27562766, 2007

[5] C. Zhai, "Positive solutions for semi-positone three-point boundary value problems," Journal of Computational and Applied Mathematics, vol. 228, no. 1, pp. 279-286, 2009.

[6] Y. Li and Z. Liang, "Two positive solutions to three-point singular boundary value problems," Acta Mathematica Scientia $B$, vol. 31, no. 1, pp. 29-38, 2011.

[7] B. Sun, A. Yang, and W. Ge, "Successive iteration and positive solutions for some second-order three-point $p$-Laplacian boundary value problems," Mathematical and Computer Modelling, vol. 50, no. 3-4, pp. 344-350, 2009.

[8] F. J. Torres, "Positive solutions for a mixed-order three-point boundary value problem for $p$-Laplacian," Abstract and Applied Analysis, vol. 2013, Article ID 912576, 8 pages, 2013.

[9] A. J. B. Potter, "Applications of Hilbert's projective metric to certain classes of non-homogeneous operators," The Quarterly Journal of Mathematics, vol. 28, no. 109, pp. 93-99, 1977.

[10] H. Amann, "Fixed point equations and nonlinear eigenvalue problems in ordered Banach spaces," SIAM Review, vol. 18, no. 4, pp. 620-709, 1976.

[11] D. J. Guo, "Fixed points and eigenelements of a class of concave and convex operators," Chinese Science Bulletin, vol. 30, no. 15, pp. 1132-1135, 1985.

[12] F. Y. Li and Z. D. Liang, "Fixed points theoremfor $\varphi$-concave (convex) operator with applications," Journal of System Science and Mathematical Science Chinese Series, vol. 14, no. 4, pp. 335360, 1994.

[13] W. X. Wang and Z. D. Liang, "Fixed point theorems for a class of nonlinear operators and their applications," Acta Mathematica Sinica, vol. 48, no. 4, pp. 789-800, 2005. 
[14] C. Zhai and D. R. Anderson, "A sum operator equation and applications to nonlinear elastic beam equations and LaneEmden-Fowler equations," Journal of Mathematical Analysis and Applications, vol. 375, no. 2, pp. 388-400, 2011.

[15] C.-B. Zhai, C. Yang, and C.-M. Guo, "Positive solutions of operator equations on ordered Banach spaces and applications," Computers \& Mathematics with Applications, vol. 56, no. 12, pp. 3150-3156, 2008.

[16] D. J. Guo and V. Lakshmikantham, Nonlinear Problems in Abstract Cones, vol. 5, Academic Press, New York, NY, USA, 1988.

[17] K. Deimling, Nonlinear Functional Analysis, Springer, Berlin, Germany, 1985. 


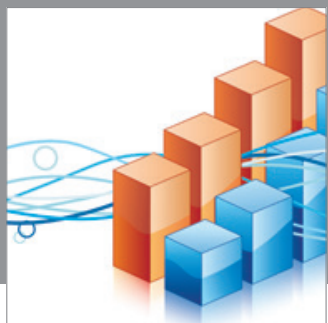

Advances in

Operations Research

mansans

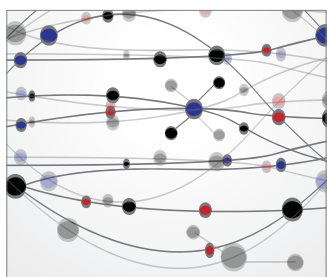

The Scientific World Journal
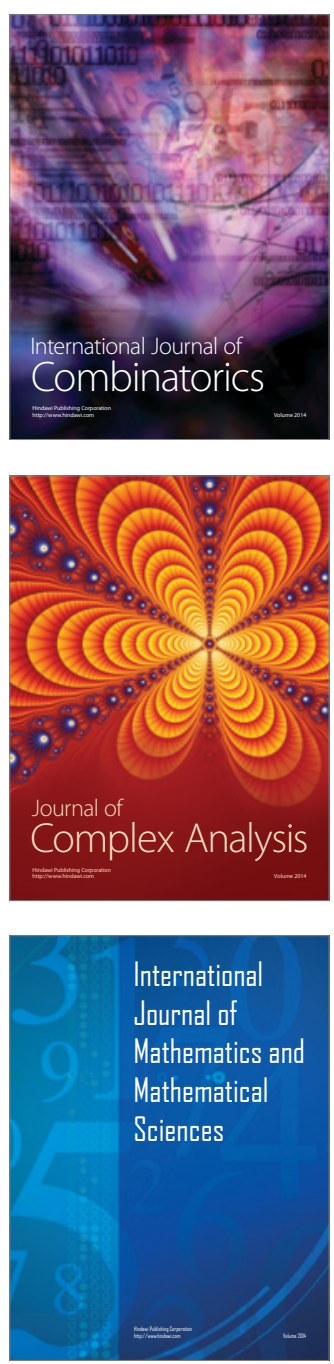
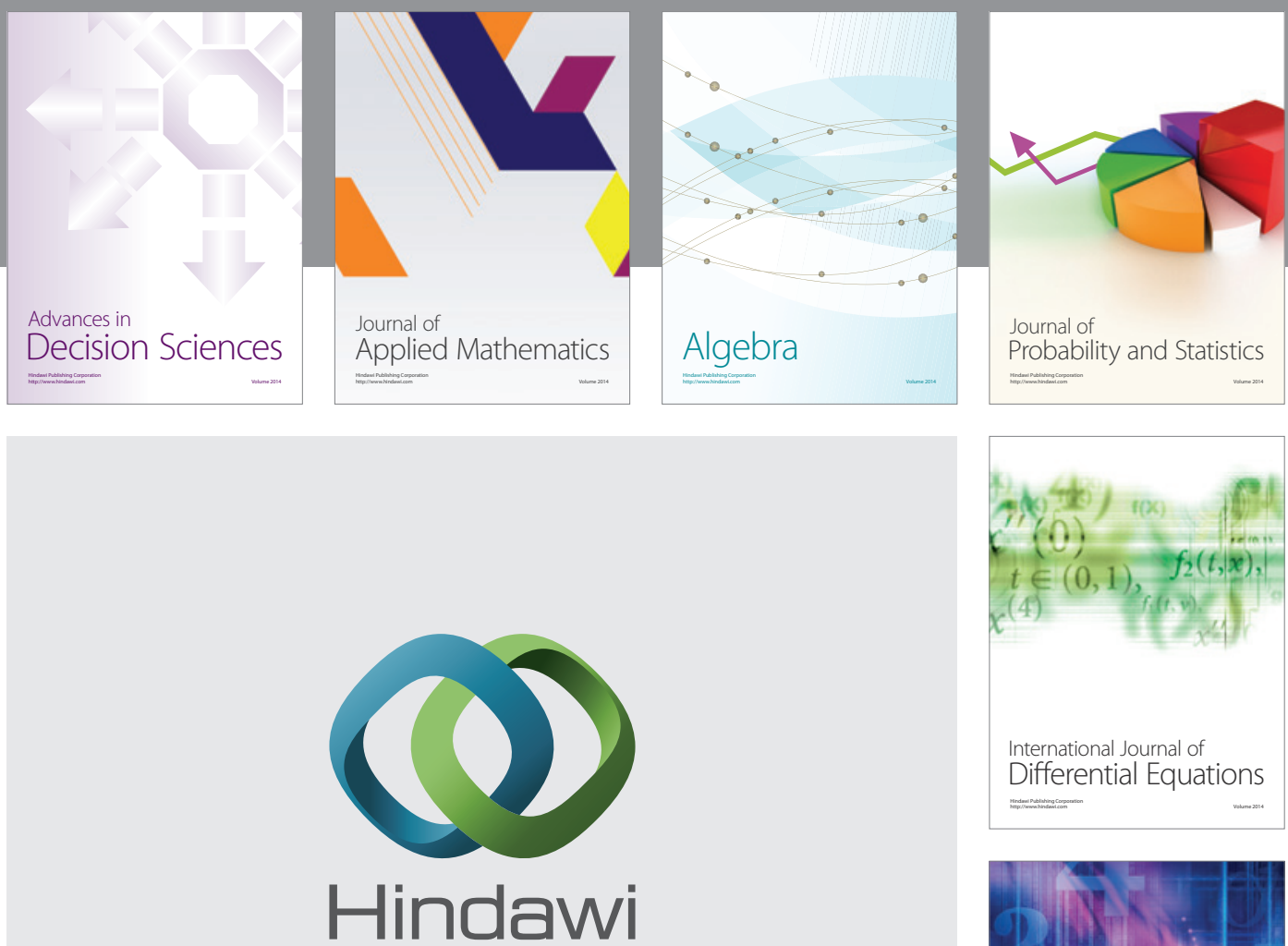

Submit your manuscripts at http://www.hindawi.com
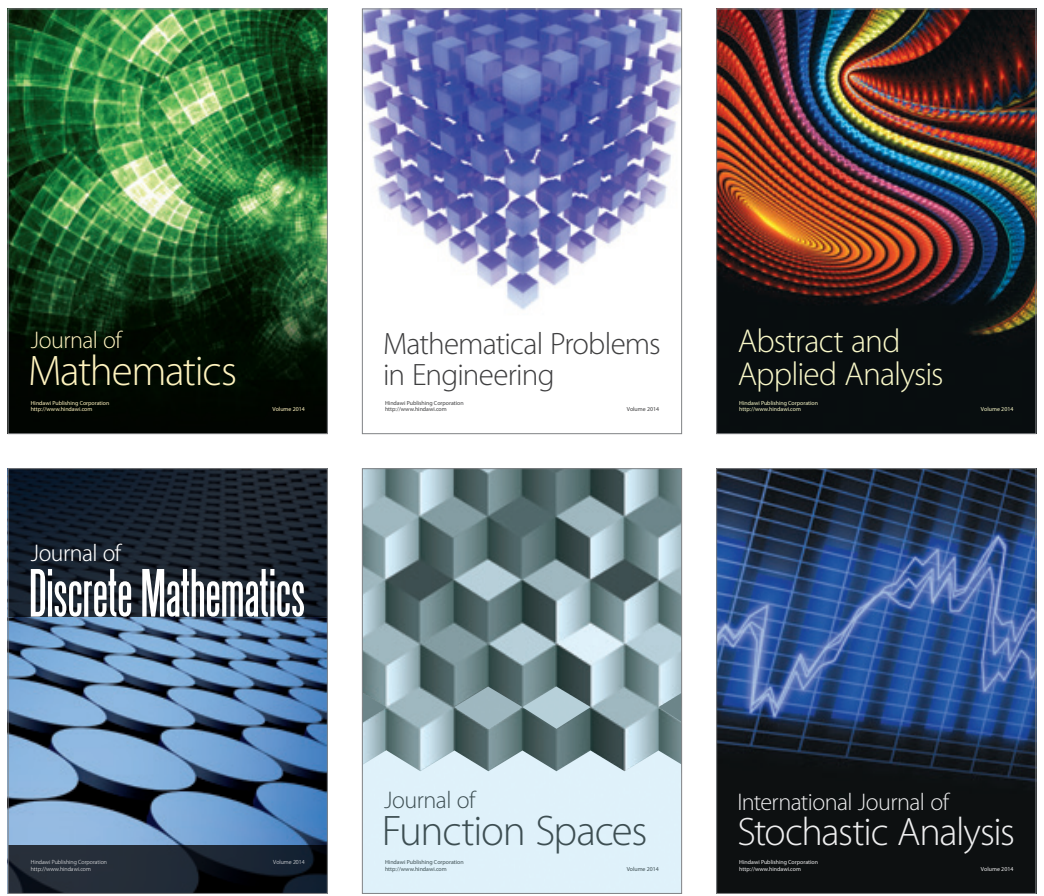

Journal of

Function Spaces

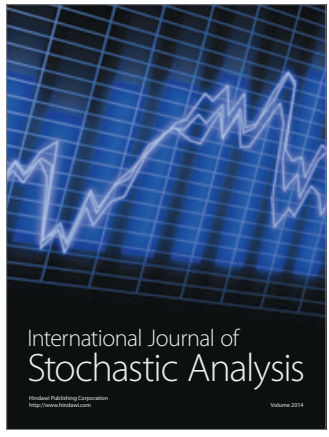

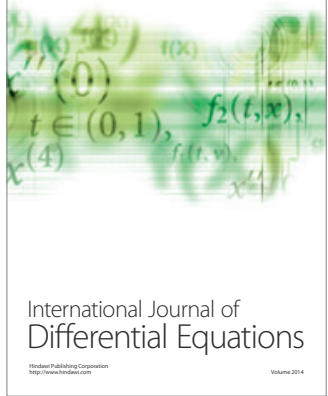
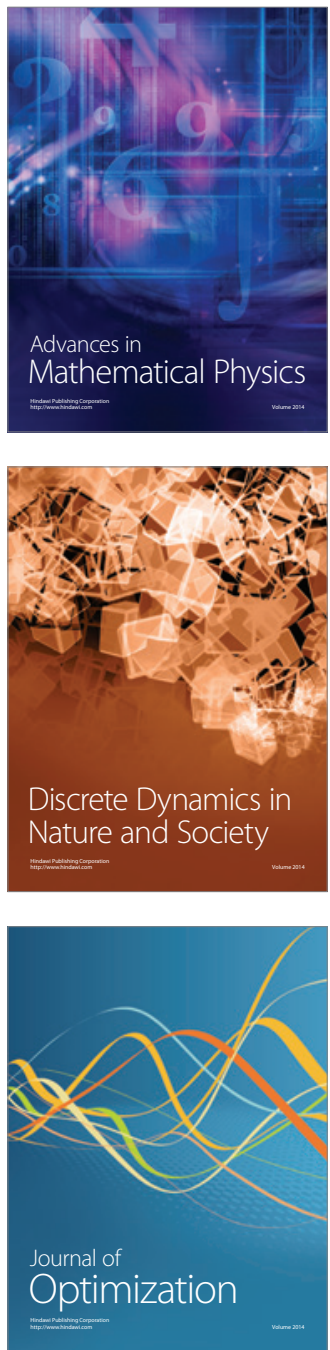